\title{
The effect of weekly specialist palliative care teleconsultations in patients with advanced cancer -a randomized clinical trial
}

Patrick D. Hoek ${ }^{1 *}$, Henk J. Schers ${ }^{2}$, Ewald M. Bronkhorst ${ }^{3}$, Kris C. P. Vissers ${ }^{1}$ and Jeroen G. J. Hasselaar ${ }^{1}$

\begin{abstract}
Background: Teleconsultation seems to be a promising intervention for providing palliative care to home-dwelling patients; however, its effect on clinically relevant outcome measures remains largely unexplored. Therefore, the purpose of this study was to determine whether weekly teleconsultations from a hospital-based specialist palliative care consultation team (SPCT) improved patient-experienced symptom burden compared to "care as usual". Secondary objectives were to determine the effects of these teleconsultations on unmet palliative care needs, continuity of care, hospital admissions, satisfaction with teleconsultations, and the burden experienced by informal caregivers.

Methods: Seventy-four home-dwelling patients diagnosed with advanced cancer were recruited from outpatient clinics of a tertiary university hospital and from regional home care organizations between May 2011 and January 2015. Participants were randomized to receive weekly, prescheduled teleconsultations with an SPCT-member (intervention group), or to receive "care as usual" (control group), for a period of 12 weeks. The primary outcome of this study was: patient-experienced symptom burden indicated by the following: (1) Total Distress Score (defined as the sum of all nine subscales of the Edmonton Symptom Assessment System) and (2) the Hospital Anxiety and Depression Scale. Mixed models were used to test for differences between the two groups.

Results: The Total Distress Score became significantly higher in the intervention group than in the control group, reaching significance at week 12 (adjusted difference at week 12: 6.90 points, 95\% Cl, 0.17 to 13.63; $P=0.04$ ). The adjusted anxiety scores were higher in the intervention group than in the control group (estimate effect: 1.40; $95 \% \mathrm{Cl}$, 0.14 to $2.55 ; P=0.03$ ). No difference was found between the groups in adjusted depression scores (estimate effect: 0.30 ; $95 \% \mathrm{Cl},-1.39$ to $1.99 ; P=0.73$ ) or in secondary outcome measures.
\end{abstract}

Conclusions: Adding weekly teleconsultations to usual palliative care leads to worse reported symptom scores among home-dwelling patients with advanced cancer. Possible explanations for these findings include excess attention on symptoms and (potential) suffering, the supply-driven care model for teleconsultations used in this trial, and the already high level of specialist palliative care provided to the control group in this study.

Trial registration: "The Netherlands National Trial Register", NTR2817, prospectively registered: March 21, 2011.

Keywords: Telemedicine, Teleconsultations, Advanced cancer, Palliative care, Symptom burden

\footnotetext{
* Correspondence: patrick.hoek@radboudumc.nl

'Department of Anesthesiology, Pain Medicine and Palliative Care, Radboud

university medical center, Nijmegen, The Netherlands

Full list of author information is available at the end of the article
} 


\section{Background}

Palliative care intends to improve the quality of life of patients facing life-threatening illnesses and that of their families [1]. Due to an increase in the number of patients dying from chronic, life-threatening conditions, the need for palliative care is expected to rise $[2,3]$. In the Netherlands, as well as in other Western countries, the majority of these patients prefer to be cared for at home until death [4, 5]. An important condition for dying at home is the availability of easily accessible, communitybased palliative home care [6-9]. Consequently, general practitioners (GPs) play a vital role in the delivery of palliative care to home-dwelling patients $[8,10]$.

However, when a patient's condition deteriorates, palliative care can become increasingly complex [11]. As a result, GPs may require additional expertise [12-14]. Sustainable models for collaboration between GPs and expert palliative care teams should therefore be developed to guarantee proactive, continuous, yet patient-centered palliative home care. Telemedicine might be an innovative approach to supporting these collaborations [15].

One of the applications of telemedicine is videoconferencing (also video- or teleconsultations), which involves the use of real-time (synchronous) audio-visual communication technology [16]. Videoconferencing has been proven to be feasible, acceptable, and effective in different fields of medicine, including psychiatry, diabetes care, and oncology [17-20]. Within palliative care, videoconferencing techniques have been used to establish multidisciplinary meetings between (rural) health centers and specialized institutes, to support patients and their families in their home-environment, and to deliver hospice care to patients living at a distance [21-26]. The results regarding the use of these techniques in palliative care are promising in terms of feasibility, acceptance and satisfaction among its users, cost-efficacy, and quality of care [22-27].

Although these results are promising, studies describing the use of videoconferencing techniques to improve the quality of palliative care for patients residing at home are generally small or have methodological limitations [16]. Recent reviews on this topic emphasize the need for randomized clinical trials (RCTs) with clinically relevant outcomes that are measured with validated instruments $[16,28-30]$.

Therefore, the primary objective of this study was to evaluate whether weekly teleconsultations between patients receiving palliative home care and a hospital-based specialist palliative care consultation team (SPCT) improves patient-experienced symptom burden when compared to "care as usual".

The secondary objectives were to evaluate the effect of these teleconsultations on (1) unmet palliative care needs, (2) experienced continuity of medical care, (3) hospital admissions, and (4) satisfaction with teleconsultations.
Furthermore, the effect of teleconsultations on the burden experienced by informal caregivers, as well as healthcare professionals' satisfaction with teleconsultations, were evaluated.

\section{Methods \\ Design}

We conducted a two-armed, non-blinded randomized clinical trial. The study protocol (Reg 2010/382) was approved by the Committee on Research Involving Human Subjects Region Arnhem-Nijmegen and has been previously published [15]. The study was prospectively registered at The Netherlands National Trial Register (NTR2817). During the study period, two amendments were approved by the Committee on Research Involving Human Subjects. Written informed consent was obtained from all participants.

\section{Participants}

\section{Setting and location}

Initially, the inclusion period for this trial was 18 months. However, mainly as a result of recruitment delay, the inclusion period was extended to 45 months (May 2011-January 2015). Patients were recruited from the outpatient clinics of the Radboud university medical center, Nijmegen, the Netherlands, mainly at the Department of Palliative Medicine as well as from regional home care organizations.

\section{Inclusion criteria}

Participants had to be aged 18 years or above, Dutch speaking, and able to give informed consent. Furthermore, they initially had to meet the following criteria: (1) be diagnosed with a progressive oncological condition, (2) reside at home, (3) have a GP who agrees to participate, (4) have a Karnofsky Performance Status score (KPS) of 60 or below, and (5) have a life-expectancy of 3 months or less.

Because of recruitment problems, the first amendment was implemented in February 2013, after 24 participants had been included. The criteria of a KPS of 60 or below and life expectancy of 3 months or less were replaced by a new criterion, namely that patients should not be receiving any disease-modifying treatment at the time of inclusion nor would do so in the future. However, as recruitment did not improve sufficiently, a second amendment was implemented in July 2013, after 35 participants had been included, whereby the latter criterion was removed and was not replaced with new inclusion criteria.

\section{Randomization}

After completing the baseline measurements, the participants were randomized into two groups: intervention or control. We used block randomization with different size blocks (4 and 6) to maintain an equal balance between groups. Randomization occurred at the level of individual patients, with an allocation ratio of 1:1. The author 
involved in the process of approaching, informing and visiting participants $(\mathrm{PH})$ was not informed about the outcome of the randomization process before baseline measurements had taken place.

Initially, it was expected that patients would be recruited by their GPs and therefore, to prevent bias, a cluster randomization procedure at the level of the GP was described in the original study protocol [15]. However, the vast majority of patients were eventually recruited via the SPCT and GPs did not recruit individual participants for this study. Consequently, there were no clusters of participants with the same GP. Therefore, in the first amendment, we decided that there was no further need for cluster randomization. As a result, randomization took place at the level of the individual participant.

\section{Care as usual}

Participants in both groups received palliative home care provided by their GP, supported by the SPCT according to the standard referral procedures, i.e., patients could be referred to the SPCT by their GP or by the attending hospital specialist or were not referred at all. If applicable, follow-up by the SPCT occurred by phone or by patients visiting the outpatient clinic, depending on the patient's preference, the complexity of their problems, and/or the stage of their disease.

\section{Intervention group \\ Procedure}

Participants in the intervention group had weekly teleconsultations for a period of 13 weeks in addition to their usual care. First, a teleconsultation device was installed at the patient's home. Patients who had not visited the SPCT before were evaluated at the outpatient clinic or during a home visit by one of the SPCT members (a nurse or physician). Then, teleconsultations were scheduled on a weekly basis for a period of 13 weeks. At the agreed time, a member of the SPCT (mostly the nurse practitioner) initiated the teleconsultation. In between these scheduled appointments, the participants could not directly contact the SPCT members through teleconsultation. When in need of medical advice, the patients were encouraged to contact their GP; however, if necessary, the SPCT could be reached by phone. A predefined consultation schedule was available to the SPCT members to ensure that all domains of palliative care were sufficiently covered during the teleconsultations. Problems and needs of participants were identified and discussed with other team members if necessary. The participant's GP was invited to visit and join the patient during the teleconsultation appointments. If this was not possible, after the first teleconsultation, a member of the SPCT contacted the patient's GP by phone to discuss the patient's current problems and needs, possible treatment policies, and the GP's involvement during the following study period. After the first and the last scheduled teleconsultation, the SPCT was encouraged to send a letter to the participant's GP, outlining the patient's current problems and needs and advised treatment policies.

\section{Teleconsultation device}

Initially, in 2011, teleconsultation devices consisted of a Pal4-desktop computer ("Bidibox", Focuscura Inc., the Netherlands) with a touch screen, a separate microphone/ speaker, and a separate camera. During the study period, tablet computers became available. These devices seemed more user-friendly, and therefore the "Bidibox" computers were replaced by tablet computers (iPad $2^{\circ}$ and iPad mini ${ }^{\circ}$; Apple Inc., United States). The Pal4 application was replaced by FaceTime ${ }^{\circ}$ (Apple Inc., United States).

\section{Outcome measures}

The primary outcome was patient-experienced symptom burden, based on the Edmonton Symptom Assessment System (ESAS) and the Hospital Anxiety and Depression Scale (HADS). The secondary and other study outcomes have been described in our study protocol [15].

\section{Data collection}

Data collection ended after 12 weeks. During the 13th week of the study period a closing teleconsultation was scheduled for participants from the intervention group. During this 13th week participants did not complete any questionnaires.

Questionnaires for baseline measurements were handed over by one of the researchers $(\mathrm{PH})$ or sent through postal mail. After completion, participants handed over the questionnaires to the researchers or sent them back through postal mail. During the study period, participants received and returned the required questionnaires through postal mail and sent them back every 4 weeks. If necessary, one of the researchers $(\mathrm{PH})$ reminded the participants by phone, SMS, or e-mail to return the required questionnaires.

Participants completed the following questionnaires: the ESAS (at baseline, weekly follow-up), HADS, Problems and Needs in Palliative Care-short version, and a modified version of the Nijmegen Continuity Questionnaire (NCQ) (all three: at baseline, four-weekly follow-up). Informal caregivers completed one questionnaire: Self-Perceived Burden from Informal Care (EDIZ) (at baseline, two-weekly follow-up).

Additionally, participants in the intervention group completed a Patient Satisfaction Questionnaire (PSQ) after the first two teleconsultations. If applicable, the SPCT members involved in the teleconsultations and the participant's GPs also completed a PSQ after the first two teleconsultations.

Finally, demographic information was collected at baseline. Information on other study outcomes (GP contacts, complex interventions, and hospital admissions [15]) was requested from the patient's GP after the study period. 


\section{Questionnaires}

The ESAS is a self-reporting scale consisting of nine symptoms that are common in patients diagnosed with cancer [31]. Items can be scored on a 0-10 visual numerical scale (with 0 indicating the absence of a symptom and 10 indicating the worst imaginable intensity of a symptom). The ESAS is widely used, and its psychometric properties are considered good in our study population [32-35]. The Total Distress Score (TDS) is defined as the sum of the nine subscales. The HADS is a 14-item self-report screening scale that provides an indication of the possible presence of anxiety and depressive symptoms [36]. Each item is scored on a 4-point Likert scale. The questions assess symptoms in the preceding week. Its psychometric properties are considered moderate to good [35, 37].

The secondary outcomes of this study were measured by the following questionnaires: (1) the Problems and Needs in Palliative Care-short version (patient-experienced problems and needs) [38, 39], (2) the NCQ (patientexperienced continuity of care) [40, 41], (3) the PSQ (satisfaction with teleconsultations) [42-44], and (4) the Self-Perceived Burden from Informal Care [45].

\section{Sample size}

For the original calculation of sample size, we refer to our study protocol [15]. As a result of the aforementioned changes in the randomization procedure, we removed the correction factor to adjust for a cluster effect, yielding an aimed total sample size of 84 patients.

\section{Statistical analysis}

Data were stored and analyzed in the Radboud university medical center, Nijmegen, the Netherlands, using SPSS Software (IBM Corp. Released 2011. IBM SPSS Statistics for Windows, Version 20.0. Armonk, NY: IBM Corp).

Observed values were reported as the mean and standard deviation (SD) for continuous variables, and the number and percentage for categorical variables.

The null hypothesis for this study was that there would be no difference in patient-experienced symptom burden between the intervention and control groups. To test this hypothesis, for all outcomes, a mixed model with a random intercept for "Patient" was used to accommodate the repeated measurements over time. The dependent variable was the relevant outcome, at any time after T0. For scale variables, a linear mixed model was used, while for dichotomous outcomes, a generalized mixed model with a logit-link function was used. To identify the best model, a series of models was tested. Starting from the simplest model, each subsequent model was extended step by step until further extensions showed non-significant improvement.

The simplest model had only the Experimental condition (i.e., "group": intervention or control) and the measurement of the outcome variable at T0 ("score at baseline") as the independent variables. The next step added Time as an independent variable. After that, the interaction between Experimental condition and Time ("group*time") was considered.

By using mixed models, every available observation contributes to the modeling of the relation between outcome and variables. As a result of this approach, missing data did not result in exclusion of participants from analyses.

Statistical software R, version 3.0.1, was used in combination with the lmer procedure from the lme 4 library for the mixed modeling analyses.

\section{Results}

A total of 957 patients were screened. We excluded patients who (1) did not meet the inclusion criteria $(n=511)$; (2) were not approached for participation for other reasons $(n=202)$; or (3) were not willing to participate $(n=167)$. Thus, a total of 77 participants gave informed consent and were enrolled in the study. Of these participants, three did not complete the baseline measurement. Consequently, 74 participants were randomized to either the intervention group $(n=38)$ or the control group $(n=36)$. A total of 32 participants $(42 \%)$ completed the study. All other participants prematurely ended this study for several reasons, mostly related to death or deteriorating condition (Fig. 1). The attrition rates were relatively high in both groups; $61 \%$ and $53 \%$ of participants in the intervention and control group, respectively, stopped contributing data during the course of the study. These rates did not differ between groups $(P=0.64)$. Sensitivity analyses on the attrition rates in relation to baseline measurements of participants showed a significant correlation between baseline scores on TDS, HADS-anxiety, and HADS-depression and attrition rates for participants from the control group. The effect of this on the study outcomes was mitigated by including baseline scores as independent variables in the regression models.

Demographic baseline characteristics are shown in Table 1. All 74 participants were analyzed for the primary outcome. Furthermore, 71 participants had an informal caregiver who gave informed consent and was enrolled in the study.

Due to financial and time constraints this study was ended after 45 months before the calculated sample size was reached.

\section{Symptom burden}

At baseline, the mean observed TDS in the intervention group was almost 7 points higher $(31.03 \pm 17.21)$ than that of the control group $(24.33 \pm 14.54)$. Over the study period, the control group showed a slight decline in the mean observed TDS. The intervention group also showed a decline in the mean observed TDS during the first 8 weeks of the study; however, in the last 4 weeks, the mean TDS increased almost 9 points to $36.62( \pm 20.14)$ at week 12 , compared to 


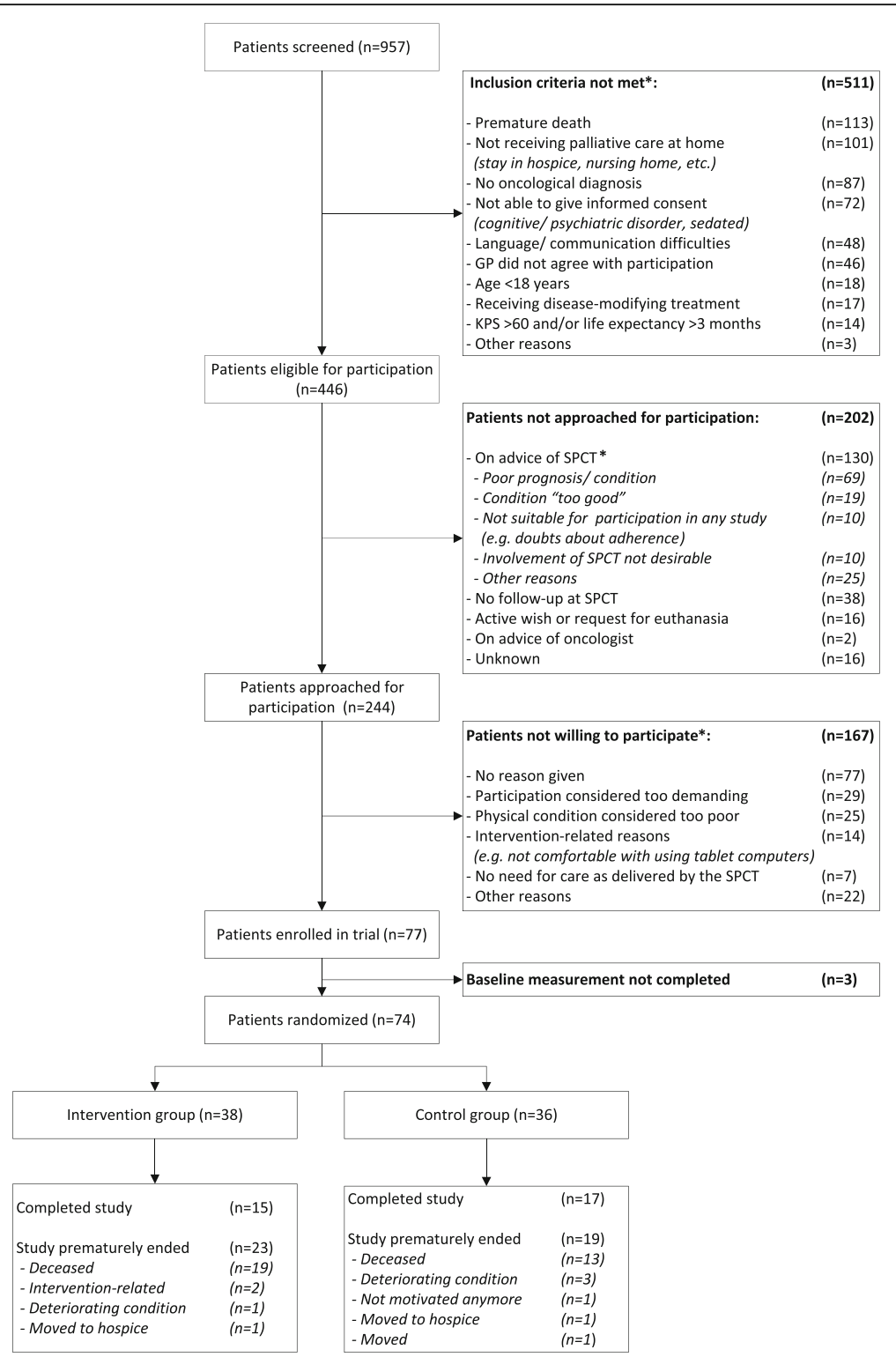

Fig. 1 Screening and participants, *More than one reason may apply

$22.38( \pm 11.27)$ in the control group (Table 2$)$. The adjusted TDS scores became significantly higher in the intervention group, indicating a growing symptom burden in this group over time compared to the control group (Fig. 2). When testing specific points in time, this difference reached significance at week 12 (adjusted difference at week 12: 6.90; 95\% CI, 0.17 to $13.63 ; P=0.04$; Table 3 ).

The mean observed HADS-anxiety scores in the control group declined somewhat during the first 4 weeks of the study (from $6.22 \pm 3.91$ to $5.23 \pm 3.41$ ) and thereafter remained rather stable. Within the intervention group, the mean observed anxiety scores were relatively stable during the first 8 weeks of the study, but increased between week 8 and week 12 from $7.11( \pm 3.39)$ to 8.46 $( \pm 4.25)$. Within the intervention group, the mean observed HADS-depression scores remained stable during the study period. The mean observed depression scores declined in the control group during the first 8 weeks (from $6.49 \pm 4.57$ to $5.76 \pm 3.92$ ); however, over the last 4 weeks, depression scores increased to $7.00( \pm 4.95)$ at week 12 (Table 2 ). The adjusted anxiety scores were significantly higher in the intervention group than in the control group (estimate effect: 1.40 ; $95 \%$ CI, 0.14 to 2.66; $P=0.03)$. The depression scores did not differ between the two groups (estimate effect: $0.30 ; 95 \% \mathrm{CI},-1.39$ to 1.99; $P=0.73$; Table 3 ). 
Table 1 Baseline characteristics of the participants $(n=74)$

\begin{tabular}{|c|c|c|c|c|}
\hline Demographics & \multicolumn{2}{|c|}{$\begin{array}{l}\text { Intervention } \\
\text { group }(n=38)\end{array}$} & \multicolumn{2}{|c|}{$\begin{array}{l}\text { Control group } \\
(n=36)\end{array}$} \\
\hline Age, mean (SD), years & 62.3 & $(9.0)$ & 61.9 & $(10.6)$ \\
\hline Female, n (\%) & 11 & (29) & 14 & (39) \\
\hline \multicolumn{5}{|l|}{ Diagnosis, n (\%) } \\
\hline Urogenital cancer & 13 & (34) & 15 & (42) \\
\hline Gastro-intestinal cancer & 6 & (16) & 5 & (14) \\
\hline Hepatobiliary and pancreatic cancer & 4 & (11) & 5 & (14) \\
\hline Lung cancer & 6 & (16) & 2 & (6) \\
\hline Head and neck cancer & 3 & (8) & 5 & (14) \\
\hline Breast cancer & 3 & (8) & 0 & (0) \\
\hline Skin cancer & 0 & (0) & 1 & (3) \\
\hline Other type of cancer & 3 & (8) & 3 & (8) \\
\hline \multicolumn{5}{|l|}{ Marital status, n (\%) } \\
\hline Married/permanent relationship & 27 & (71) & 29 & (81) \\
\hline Divorced & 5 & (13) & 1 & (3) \\
\hline Single & 4 & (11) & 5 & (14) \\
\hline Widow(er) & 2 & (5) & 1 & (3) \\
\hline Having one or more children, $\mathrm{n}(\%)$ & 32 & (84) & 30 & (83) \\
\hline \multicolumn{5}{|l|}{ Living situation, n (\%) } \\
\hline Together with partner and/or children & 28 & (74) & 29 & (81) \\
\hline Alone & 9 & (24) & 6 & (17) \\
\hline Other living situation & 1 & (3) & 1 & (3) \\
\hline Household, mean number of persons (SD) & 2.0 & $(0.9)$ & 2.0 & $(0.6)$ \\
\hline \multicolumn{5}{|l|}{ Highest educational level, n (\%) } \\
\hline No education/primary school & 1 & (3) & 3 & (8) \\
\hline Lower vocational education & 10 & (26) & 7 & (19) \\
\hline Lower general secondary education & 4 & (11) & 8 & (22) \\
\hline Intermediate vocational education & 11 & (29) & 7 & (19) \\
\hline $\begin{array}{l}\text { Higher general secondary education/ } \\
\text { pre-university education }\end{array}$ & 3 & (8) & 2 & (6) \\
\hline Higher professional education/university & 9 & (24) & 9 & (25) \\
\hline
\end{tabular}

Percentages may not add to $100 \%$ due to rounding

SD Standard deviation

\section{Secondary and other study outcomes}

The mean number of unmet needs did not differ between the intervention and control groups (estimate effect: -0.01 ; $95 \% \mathrm{CI},-0.07$ to $0.04 ; P=0.67$ ). Additionally, the number of participants having at least one unmet need did not differ between the groups (OR: 0.79; $95 \% \mathrm{CI}, 0.19$ to $2.92 ; P=$ 0.66). On all three subscales of the NCQ for continuity of care, i.e., personal continuity (estimate effect: $0.15 ; 95 \% \mathrm{CI}$, -0.09 to $0.38 ; P=0.22$ ), team continuity (estimate effect: 0.16 ; $95 \% \mathrm{CI},-0.20$ to $0.51 ; P=0.39$ ), and cross-boundary continuity (estimate effect: $0.29 ; 95 \% \mathrm{CI},-0.08$ to 0.67 ; $P=0.13$ ), there were no differences between groups. Finally, the mean number of hospital admissions during the study period did not differ between the intervention group (0.47) and the control group (0.38; $P=0.60)$. Study outcome measures regarding GP contacts and complex interventions did not statistically differ between both groups. The mean satisfaction scores after the first two teleconsultations were high for both the participants $(90.4 \pm 8.2$ and $89.4 \pm 9.7)$ and the SPCT members $(87.0 \pm 7.1$ and $85.5 \pm 15.2)$.

When comparing the number of informal caregivers with a high perceived burden (defined as the upper third of the group), there was a trend towards a lower proportion of informal caregivers with a high perceived burden in the intervention group (estimate effect: $-2.24 ; 95 \% \mathrm{CI}$, -5.24 to $0.02 ; P=0.05$ ).

\section{Discussion}

Main results

We found a difference in reported symptom burden between home-dwelling patients with advanced cancer receiving palliative care "as usual" and patients who additionally had weekly teleconsultations with a hospital-based SPCT. Therefore, we can reject our null hypothesis, which stated that there would be no difference in patient-experienced symptom burden between the intervention group and the control group. Contrary to our expectations, this additional intervention led to a higher reported symptom burden in the intervention group than in the control group. The number of unmet needs, experienced continuity of care, and reported hospital admissions did not differ between groups.

\section{Comparison with the literature}

To the best of our knowledge, this is the first RCT to show that, despite difficulties in recruitment, technical challenges, and relatively high drop-out rates, it is possible to perform an RCT on telemedicine, which can be considered a complex intervention, in palliative care [46].

We found an observed mean TDS ranging from 21.4 to 36.6. This is comparable to other studies reporting ESAS scores from patients with advanced cancer visiting outpatient clinics [47-49], although higher scores have been reported [50].

While the TDS in the control group remained relatively stable during the study period, the TDS of the intervention group increased. For both the observed and the adjusted TDS, this increase was of more than 4 points, indicating a clinically relevant deterioration according to Hui et al. [49]. This deterioration seems to be in contrast with earlier research in which video technologies did not affect symptom burden or even led to a possible improvement in clinical outcomes $[21,25]$. On the other hand, the adjusted difference between both groups at week 12 was less than 8 points, which is the minimum clinically important difference stated in the power calculation of our study protocol [15]. Therefore, and given the relatively wide confidence intervals of the 
Table 2 Observed values at baseline and weeks 4, 8, and 12

\begin{tabular}{|c|c|c|c|c|c|c|}
\hline & & Group & Baseline & Week 4 & Week 8 & Week 12 \\
\hline \multicolumn{7}{|l|}{ ESAS } \\
\hline \multirow[t]{2}{*}{ TDS, mean (SD) } & $(0-90)$ & Intervention & $\begin{array}{l}31.03(17.21) \\
n=38\end{array}$ & $\begin{array}{l}30.68(19.58) \\
n=22\end{array}$ & $\begin{array}{l}27.73(15.87) \\
n=15\end{array}$ & $\begin{array}{l}36.62(20.14) \\
n=13\end{array}$ \\
\hline & & Control & $\begin{array}{l}24.33 \\
n=36\end{array}$ & $\begin{array}{l}24.17(13.79) \\
n=27\end{array}$ & $\begin{array}{l}22.20 \\
\mathrm{n}=20\end{array}$ & $\begin{array}{l}22.38(11.27) \\
n=16\end{array}$ \\
\hline \multicolumn{7}{|l|}{ HADS } \\
\hline \multirow[t]{2}{*}{ Anxiety, mean (SD) } & $(0-21)$ & Intervention & $\begin{array}{l}7.24(4.70) \\
n=38\end{array}$ & $\begin{array}{l}7.48(4.19) \\
\mathrm{n}=23\end{array}$ & $\begin{array}{l}7.11(3.39) \\
n=16\end{array}$ & $\begin{array}{l}8.46(4.25) \\
n=13\end{array}$ \\
\hline & & Control & $\begin{array}{l}6.22(3.91) \\
n=36\end{array}$ & $\begin{array}{l}5.23(3.41) \\
n=26\end{array}$ & $\begin{array}{l}4.71(3.08) \\
n=21\end{array}$ & $\begin{array}{l}5.06(3.21) \\
n=16\end{array}$ \\
\hline \multirow[t]{2}{*}{ Depression, mean (SD) } & $(0-21)$ & Intervention & $\begin{array}{l}7.66(3.87) \\
n=38\end{array}$ & $\begin{array}{l}7.45(4.82) \\
n=23\end{array}$ & $\begin{array}{l}7.31(4.45) \\
n=16\end{array}$ & $\begin{array}{l}7.85(5.10) \\
n=13\end{array}$ \\
\hline & & Control & $\begin{array}{l}6.49(4.57) \\
n=36\end{array}$ & $\begin{array}{l}6.25(4.16) \\
n=26\end{array}$ & $\begin{array}{l}5.76(3.92) \\
n=21\end{array}$ & $\begin{array}{l}7.00(4.95) \\
n=16\end{array}$ \\
\hline \multicolumn{7}{|l|}{ PNPC-sV } \\
\hline \multirow[t]{2}{*}{ Number of unmet needs, mean (SD) } & $(0-32)$ & Intervention & $\begin{array}{l}3.94(5.68) \\
n=37\end{array}$ & $\begin{array}{l}2.07(3.82) \\
n=23\end{array}$ & $\begin{array}{l}1.31 \\
n=16\end{array}$ & $\begin{array}{l}2.02(3.88) \\
n=13\end{array}$ \\
\hline & & Control & $\begin{array}{l}2.92(4.36) \\
n=36\end{array}$ & $\begin{array}{l}2.57(3.75) \\
n=25\end{array}$ & $\begin{array}{l}1.42(2.60) \\
n=17\end{array}$ & $\begin{array}{l}2.80(5.21) \\
n=15\end{array}$ \\
\hline \multicolumn{7}{|l|}{ NCQ } \\
\hline \multirow[t]{2}{*}{ Personal continuity, mean (SD) } & $(6-30)$ & Intervention & $\begin{array}{l}24.33(3.76) \\
n=36\end{array}$ & $\begin{array}{l}24.52(3.10) \\
n=23\end{array}$ & $\begin{array}{l}24.73(3.26) \\
n=15\end{array}$ & $\begin{array}{l}24.38(3.55) \\
n=13\end{array}$ \\
\hline & & Control & $\begin{array}{l}22.81 \\
\mathrm{n}=33\end{array}$ & $\begin{array}{l}23.00(3.43) \\
n=25\end{array}$ & $\begin{array}{l}23.28 \\
n=18.33)\end{array}$ & $\begin{array}{l}21.92(4.27) \\
n=13\end{array}$ \\
\hline \multirow[t]{2}{*}{ Team continuity (within hospital), mean (SD) } & $(4-20)$ & Intervention & $\begin{array}{l}14.20(3.26) \\
n=30\end{array}$ & $\begin{array}{l}15.89(2.27) \\
n=18\end{array}$ & $\begin{array}{l}15.75 \\
n=12\end{array}$ & $\begin{array}{l}14.60(3.24) \\
n=10\end{array}$ \\
\hline & & Control & $\begin{array}{l}15.12(3.15) \\
n=31\end{array}$ & $\begin{array}{l}15.59(2.92) \\
n=17\end{array}$ & $\begin{array}{l}15.42(2.61) \\
n=12\end{array}$ & $\begin{array}{l}13.73(2.45) \\
n=11\end{array}$ \\
\hline \multirow[t]{2}{*}{ Cross-boundary continuity, mean (SD) } & $(4-20)$ & Intervention & $\begin{array}{l}15.83 \\
n=24\end{array}$ & $\begin{array}{l}16.56 \\
n=18.01)\end{array}$ & $\begin{array}{l}14.20(2.78) \\
n=15\end{array}$ & $\begin{array}{l}16.59(2.98) \\
n=13\end{array}$ \\
\hline & & Control & $\begin{array}{l}15.16(3.13) \\
n=25\end{array}$ & $\begin{array}{l}15.35(2.57) \\
n=18\end{array}$ & $\begin{array}{l}14.10(4.25) \\
n=13\end{array}$ & $\begin{array}{l}14.33(3.20) \\
n=9\end{array}$ \\
\hline \multicolumn{7}{|l|}{$\mathrm{EDIZ}$} \\
\hline \multirow[t]{2}{*}{ Total score, mean (SD) } & $(9-45)$ & Intervention & $\begin{array}{l}15.78(5.87) \\
n=36\end{array}$ & $\begin{array}{l}14.54(7.09) \\
n=21\end{array}$ & $\begin{array}{l}16.09 \\
n=16\end{array}$ & $\begin{array}{l}15.95(8.25) \\
n=12\end{array}$ \\
\hline & & Control & $\begin{array}{l}15.54(6.46) \\
n=35\end{array}$ & $\begin{array}{l}17.45 \\
n=25\end{array}$ & $\begin{array}{l}14.74(5.88) \\
n=19\end{array}$ & $\begin{array}{l}15.00(7.10) \\
n=14\end{array}$ \\
\hline
\end{tabular}

SD standard deviation, ESAS Edmonton Symptom Assessment System, TDS Total Distress Score, HADS Hospital Anxiety and Depression Scale, PNPC-sv Problems and Needs in Palliative Care-Short Version, NCQ Nijmegen Continuity Questionnaire, EDIZ self-perceived burden from informal care

adjusted difference in TDS, the clinical relevance of the difference in TDS should be interpreted with proper caution.

The higher reported symptom burden found in this study might be partially explained by the participant's perceptions of symptoms. Participants in the intervention group received weekly attention for their (potential) symptoms. This might have led to a higher awareness of symptoms among participants, leading to a worsening symptom experience. This phenomenon is referred to as the "nocebo effect" [51]. In a review on this topic, Häuser et al. [51] state that patients are "highly receptive to negative suggestion, particularly in situations perceived as existentially threatening". This negative suggestion includes focusing one's attention towards the presence of particular symptoms. The "nocebo effect" may be avoided by adapting the frequency and timing of the teleconsultations to the actual needs of the individual patient.

Another explanation for our findings might be the differential recall bias among participants. For participants in the intervention group, the reported symptom burden was strongly related to the content of their weekly teleconsultations. As a result, their symptom burden may have been registered more precisely, possibly leading to higher symptom scores. 


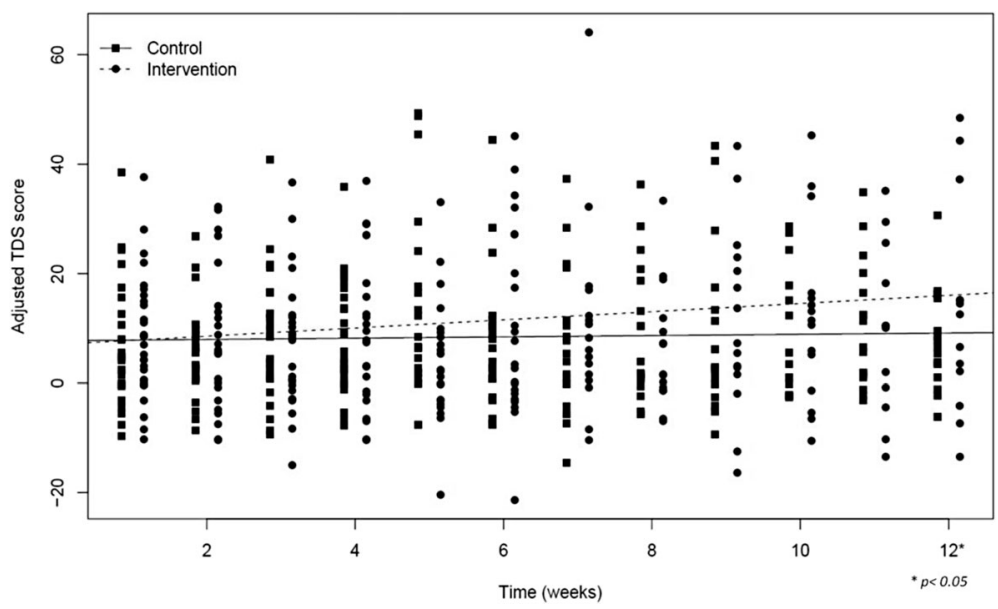

Fig. 2 Adjusted Total Distress Score during the study period

\section{Strengths and limitations}

An important strength of this study is that we have systematically screened a large group of patients for participation in this trial. Despite considerable difficulties in recruitment, the intended sample size was nearly reached, although over a longer period of time and with adjusted

Table 3 Primary outcome measures - mixed models

\begin{tabular}{|c|c|c|c|c|}
\hline & \multirow[t]{2}{*}{$B(S E)$} & \multicolumn{2}{|l|}{$95 \% \mathrm{Cl}$} & \multirow[t]{2}{*}{$P$ value } \\
\hline & & Lower limit & Upper limit & \\
\hline \multicolumn{5}{|l|}{ ESAS-TDS } \\
\hline Intercept & $7.26(3.17)$ & & & \\
\hline Group $^{a}$ & $-0.66(3.26)$ & -6.99 & 5.66 & 0.84 \\
\hline $\begin{array}{l}\text { TDS score at } \\
\text { baseline }\end{array}$ & $0.78(0.11)$ & 0.57 & 0.99 & $<0.001$ \\
\hline Time $^{b}$ & $0.12(0.17)$ & -0.21 & 0.45 & 0.48 \\
\hline Group*time & $0.63(0.25)$ & 0.14 & 1.11 & 0.01 \\
\hline \multicolumn{5}{|l|}{ HADS-A } \\
\hline Intercept & $0.54(0.76)$ & & & \\
\hline Group $^{a}$ & $1.40(0.65)$ & 0.14 & 2.66 & 0.03 \\
\hline $\begin{array}{l}\text { HADS-A score } \\
\text { at baseline }\end{array}$ & $0.78(0.09)$ & 0.61 & 0.95 & $<0.001$ \\
\hline Time $^{b}$ & $0.12(0.06)$ & 0.01 & 0.23 & 0.04 \\
\hline \multicolumn{5}{|l|}{ HADS-D } \\
\hline Intercept & $1.56(1.03)$ & & & \\
\hline Group $^{a}$ & $0.30(0.87)$ & -1.39 & 1.99 & 0.73 \\
\hline $\begin{array}{l}\text { HADS-D score } \\
\text { at baseline }\end{array}$ & $0.70(0.11)$ & 0.49 & 0.91 & $<0.001$ \\
\hline Time $^{b}$ & $0.13(0.08)$ & -0.03 & 0.29 & 0.12 \\
\hline
\end{tabular}

$B$ estimate effect, SE standard error, 95\% Cl 95\% confidence interval, ESAS Edmonton Symptom Assessment System, TDS Total Distress Score, HADS-A Hospital Anxiety and Depression Scale - Anxiety, HADS-D Hospital Anxiety and Depression Scale - Depression

${ }^{\mathrm{a}}$ Group: intervention $=1$; control $=0$

${ }^{\mathrm{b}}$ Time: week number, baseline $=$ week 0 inclusion criteria. Finally, this is the first completed RCT on telemedicine in palliative care with outcome measures that are clinically relevant and relate directly to patient care and experienced quality of life. This study also has some limitations. First, a considerable group of patients who were eligible for participation in this trial were not approached, mostly as a result of clinical considerations, which may have caused non-differential selection bias. Additionally, the relatively small number of patients who were eventually approached for participation might also reflect that offering teleconsultations in the context of a randomized study might not fit the needs of palliative care patients. Second, the attrition rate in this study was relatively high and attrition may depend on the clinical condition of participants. Although in our statistical models we have corrected for baseline measurements (i.e., the clinical condition of participants at the start of the study), study outcomes may have been influenced by participants' worsening clinical condition during the study. Third, the participants sometimes had difficulty adequately completing the questionnaires as a result of their varying clinical conditions. This might have led to information bias, although likely non-differential. Fourth, the outcome measure "place of death" was described in the study protocol, however, it was not included in the information request at the patient's GP; thus, this study outcome is missing. Fifth, two amendments had to be made to the study protocol to improve the recruitment rates. As a result of widening the inclusion criteria of this study, the study population may have become more heterogeneous, which may have led to a dilution of the effect of the intervention. Finally, the involvement of GPs in this study was less than expected; therefore, the participants were not recruited by their GP but were instead recruited at the outpatient clinic of the SPCT, probably leading to higher levels of specialist care in both groups, which may have positively affected symptom scores. 


\section{Clinical implications}

In this study, we introduced a model for teleconsultation in palliative care that was intended to be proactive and was mainly supply driven. Teleconsultations were scheduled on a weekly basis for a period of 3 months, irrespective of the actual needs of the patient regarding the timing and frequency of these teleconsultations. This model was shown to be ineffective in reducing experienced symptom burden, even though patients and caregivers showed a high degree of satisfaction. Therefore, we propose focusing on care models that are patient-tailored and demand-driven, i.e., patients themselves indicate when they are in need of palliative care (tele)consultations. This model could prevent a possible excess of medical care regarding palliative care, death, and dying. At the same time, to avoid a rather reactive palliative care approach, patients should be provided tools and support to guide them in proactively contacting their caregivers when problems arise.

\section{Conclusions}

Telemedicine is emerging in all fields of medicine, including palliative care. Despite promising earlier research, the present study shows that telemedicine does not necessarily lead to a better quality of advanced cancer care. Indeed, the use of telemedicine might create a situation in which patients experience a higher symptom burden, despite high degrees of satisfaction. Future research and care models should therefore explore the beneficial as well as the potentially harmful aspects of teleconsultations within advanced cancer care, thereby focusing on (1) ways to optimize multidisciplinary care via teleconsultations, (2) the appropriate timing and frequency of palliative care teleconsultations for patients with advanced cancer and other groups of vulnerable patients, (3) possibilities for patient-tailored, demand-driven teleconsultations, and (4) the potential impact of technology as such on the patient's sense of wellbeing. These issues should be adequately addressed, both in future research and in implementation trajectories regarding the use of telemedicine in palliative home care.

\section{Abbreviations \\ 95\% Cl: 95\% confidence interval; ESAS: Edmonton Symptom Assessment System; GP: general practitioner; HADS: Hospital Anxiety and Depression Scale; KPS: Karnofsky Performance Status score; NCQ: Nijmegen Continuity Questionnaire; PSQ: Patient Satisfaction Questionnaire; RCT: randomized clinical trial; SPCT: specialist palliative care consultation team; TDS: Total Distress Score}

\section{Acknowledgments}

The authors would like to express their gratitude to all patients and their informal caregivers who participated in this study. The authors would also like to thank the members of the SPCT for their meaningful contribution to this study.

\section{Funding}

This work was supported by Netherlands Organisation for Scientific Research (NWO). The funding organization had no role in the design of the study; collection, analysis and interpretation of the data; and in writing the manuscript.

\section{Availability of data and materials}

The datasets used and analyzed during the current study are available from the corresponding author on reasonable request.

\section{Authors' contributions}

All authors contributed substantially to this manuscript. JH, KV, HS, and $\mathrm{PH}$ were involved in the design of the study. $\mathrm{PH}$ and $\mathrm{JH}$ were involved in the acquisition of data. $\mathrm{PH}, \mathrm{JH}$ and $\mathrm{EB}$ were responsible for analysis and statistical analysis of data. $\mathrm{PH}, \mathrm{HS}, \mathrm{EB}, \mathrm{KV}$, and $\mathrm{JH}$ were responsible for interpretation of data. $\mathrm{PH}$ and $J H$ drafted the manuscript. $H S$, EB, and KV revised it critically for important intellectual content. All authors read and approved the final manuscript.

\section{Competing interests}

The authors declare that they have no competing interests.

\section{Consent for publication}

Written informed consent was obtained from all participants.

\section{Ethics approval and consent to participate}

The study protocol (Reg 2010/382) was approved by the Committee on Research Involving Human Subjects (CMO) Region Arnhem-Nijmegen. The study was prospectively registered at The Netherlands National Trial Register (NTR2817). During the study period, two amendments regarding the inclusion criteria were approved by the CMO. Written informed consent was obtained from all participants.

\section{Publisher's Note}

Springer Nature remains neutral with regard to jurisdictional claims in published maps and institutional affiliations.

\section{Author details}

'Department of Anesthesiology, Pain Medicine and Palliative Care, Radboud university medical center, Nijmegen, The Netherlands. ${ }^{2}$ Department of Primary and Community Care, Radboud university medical center, Nijmegen, The Netherlands. ${ }^{3}$ Department of Dentistry, Radboud university medical center, Nijmegen, The Netherlands.

Received: 21 November 2016 Accepted: 4 May 2017

Published online: 19 June 2017

\section{References}

1. World Health Organization. WHO Definition of Palliative Care. http://www. who.int/cancer/palliative/definition/en/. Accessed 21 Nov 2016.

2. van der Velden $L F$, Francke AL, Hingstman L, Willems DL. Dying from cancer or other chronic diseases in the Netherlands: ten-year trends derived from death certificate data. BMC Palliat Care. 2009;8:4.

3. Cohen J, Deliens L. Chapter 1: Applying a public health perspective to endof-life care. In: Cohen J, Deliens L, editors. A Public Health Perspective on End of Life Care. New York: Oxford University Press Inc.; 2012. p. 3-18.

4. Gomes B, Higginson IJ, Calanzani N, Cohen J, Deliens L, Daveson BA, Bechinger-English D, Bausewein C, Ferreira PL, Toscani F, et al. Preferences for place of death if faced with advanced cancer: a population survey in England, Flanders, Germany, Italy, the Netherlands, Portugal and Spain. Ann Oncol. 2012;23(8):2006-15.

5. Gomes B, Calanzani N, Gysels M, Hall S, Higginson IJ. Heterogeneity and changes in preferences for dying at home: a systematic review. BMC Palliat Care. 2013;12:7.

6. Gomes B, Calanzani N, Koffman J, Higginson IJ. Is dying in hospital better than home in incurable cancer and what factors influence this? A population-based study. BMC Med. 2015;13:235.

7. Quill TE, Abernethy AP. Generalist plus specialist palliative care-creating a more sustainable model. N Engl J Med. 2013;368(13):1173-5.

8. Murray SA, Firth A, Schneider N, Van den Eynden B, Gomez-Batiste X, Brogaard T, Villanueva T, Abela J, Eychmuller S, Mitchell G, et al. Promoting palliative care in the community: production of the primary palliative care toolkit by the European Association of Palliative Care Taskforce in primary palliative care. Palliat Med. 2015;29(2):101-11.

9. World Health Organization. Strengthening of Palliative Care as a Component of Comprehensive Care Throughout the Life Course. WHA Resolution; Sixty-seventh World Health Assembly. 2014. http://apps.who.int/ medicinedocs/documents/s21454en/s21454en.pdf. Accessed 15 May 2017. 
10. Groot MM, Vernooij-Dassen MJ, Crul BJ, Grol RP. General practitioners (GPS) and palliative care: perceived tasks and barriers in daily practice. Palliat Med. 2005;19(2):111-8.

11. Borgsteede SD, Deliens L, Beentjes B, Schellevis F, Stalman WA, Van Eijk JT, Van der Wal G. Symptoms in patients receiving palliative care: a study on patient-physician encounters in general practice. Palliat Med. 2007;21(5):417-23.

12. Dahlhaus A, Vanneman N, Siebenhofer A, Brosche M, Guethlin C. Involvement of general practitioners in palliative cancer care: a qualitative study. Support Care Cancer. 2013;21(12):3293-300.

13. Mitchell GK. How well do general practitioners deliver palliative care? A systematic review. Palliat Med. 2002;16(6):457-64.

14. Meijler WJ, Van Heest F, Otter R, Sleijfer DT. Educational needs of general practitioners in palliative care: outcome of a focus group study. J Cancer Educ. 2005;20(1):28-33.

15. Duursma F, Schers HJ, Vissers KC, Hasselaar J. Study protocol: optimization of complex palliative care at home via telemedicine. A cluster randomized controlled trial. BMC Palliat Care. 2011;10:13.

16. Kitamura C, Zurawel-Balaura L, Wong RK. How effective is video consultation in clinical oncology? A systematic review. Curr Oncol. 2010;17(3):17-27.

17. Himle MB, Freitag M, Walther M, Franklin SA, Ely L, Woods DW. A randomized pilot trial comparing videoconference versus face-to-face delivery of behavior therapy for childhood tic disorders. Behav Res Ther. 2012;50(9):565-70.

18. Stubbings DR, Rees CS, Roberts LD, Kane RT. Comparing in-person to videoconference-based cognitive behavioral therapy for mood and anxiety disorders: randomized controlled trial. J Med Internet Res. 2013;15(11):e258.

19. Levin K, Madsen JR, Petersen I, Wanscher CE, Hangaard J. Telemedicine diabetes consultations are cost-effective, and effects on essential diabetes treatment parameters are similar to conventional treatment: 7-year results from the Svendborg Telemedicine Diabetes Project. J Diabetes Sci Technol. 2013;7(3):587-95.

20. Seeber A, Mitterer M, Gunsilius E, Mazzoleni G, Giovannetti R, Farsad M, Eisterer W, Gastl G, Pall G, Wieser A, et al. Feasibility of a multdisciplinary lung cancer videoconference between a peripheral hospital and a comprehensive cancer centre. Oncology. 2013;84(3):186-90.

21. Hebert MA, Brant R, Hailey D, van der Pol M. Potential and readiness for video-visits in rural palliative homecare: results of a multi-method study in Canada. J Telemed Telecare. 2006;12 Suppl 3:43-5.

22. Bensink ME, Armfield NR, Pinkerton $R$, Irving $H$, Hallahan AR, Theodoros DG, Russell T, Barnett AG, Scuffham PA, Wootton R. Using videotelephony to support paediatric oncology-related palliative care in the home: from abandoned RCT to acceptability study. Palliat Med. 2009;23(3):228-37.

23. Bradford NK, Armfield NR, Young J, Smith AC. Paediatric palliative care by video consultation at home: a cost minimisation analysis. BMC Health Serv Res. 2014;14:328.

24. van Gurp J, van Selm M, Vissers K, van Leeuwen E, Hasselaar J. How outpatient palliative care teleconsultation facilitates empathic patient-professional relationships: a qualitative study. PLoS One. 2015;10(4):e0124387.

25. Watanabe SM, Fairchild A, Pituskin E, Borgersen P, Hanson J, Fassbender K. Improving access to specialist multidisciplinary palliative care consultation for rural cancer patients by videoconferencing: report of a pilot project. Support Care Cancer. 2013;21(4):1201-7.

26. Oliver DP, Demiris G, Wittenberg-Lyles E, Washington K, Day T, Novak H. A systematic review of the evidence base for telehospice. Telemed J E Health. 2012;18(1):38-47.

27. Bradford N, Young J, Armfield NR, Bensink ME, Pedersen LA, Herbert A Smith AC. A pilot study of the effectiveness of home teleconsultations in paediatric palliative care. J Telemed Telecare. 2012;18(8):438-42.

28. Kidd L, Cayless S, Johnston B, Wengstrom Y. Telehealth in palliative care in the UK: a review of the evidence. J Telemed Telecare. 2010;16(7):394-402.

29. Capurro D, Ganzinger M, Perez-Lu J, Knaup P. Effectiveness of eHealth interventions and information needs in palliative care: a systematic literature review. J Med Internet Res. 2014;16(3):e72.

30. Rogante M, Giacomozzi C, Grigioni M, Kairy D. Telemedicine in palliative care: a review of systematic reviews. Ann Ist Super Sanita. 2016:52(3):434-42.

31. Bruera E, Kuehn N, Miller MJ, Selmser P, Macmillan K. The Edmonton Symptom Assessment System (ESAS): a simple method for the assessment of palliative care patients. J Palliat Care. 1991;7(2):6-9.

32. Chang VT, Hwang SS, Feuerman M. Validation of the Edmonton Symptom Assessment Scale. Cancer. 2000;88(9):2164-71.

33. Richardson LA, Jones GW. A review of the reliability and validity of the Edmonton Symptom Assessment System. Curr Oncol. 2009;16(1):55.
34. Philip J, Smith WB, Craft P, Lickiss N. Concurrent validity of the modified Edmonton Symptom Assessment System with the Rotterdam Symptom Checklist and the Brief Pain Inventory. Support Care Cancer. 1998;6(6):539-41.

35. Aktas A, Walsh D, Kirkova J. The psychometric properties of cancer multisymptom assessment instruments: a clinical review. Support Care Cancer. 2015;23(7):2189-202

36. Zigmond AS, Snaith RP. The hospital anxiety and depression scale. Acta Psychiatr Scand. 1983;67(6):361-70.

37. Spinhoven P, Ormel J, Sloekers PP, Kempen Gl, Speckens AE, Van Hemert AM. A validation study of the Hospital Anxiety and Depression Scale (HADS) in different groups of Dutch subjects. Psychol Med. 1997;27(2):363-70.

38. Osse BH, Vernooij MJ, Schade E, Grol RP. Towards a new clinical tool for needs assessment in the palliative care of cancer patients: the PNPC instrument. J Pain Symptom Manag. 2004;28(4):329-41.

39. Osse BH, Vernooij-Dassen MJ, Schade E, Grol RP. A practical instrument to explore patients' needs in palliative care: the Problems and Needs in Palliative Care questionnaire short version. Palliat Med. 2007;21(5):391-9.

40. Uijen AA, Schellevis FG, van den Bosch WJ, Mokkink HG, van Weel C, Schers HJ. Nijmegen Continuity Questionnaire: development and testing of a questionnaire that measures continuity of care. J Clin Epidemiol. 2011;64(12):1391-9.

41. Uijen AA, Schers HJ, Schellevis FG, Mokkink HG, van Weel C, van den Bosch WJ. Measuring continuity of care: psychometric properties of the Nijmegen Continuity Questionnaire. Br J Gen Pract. 2012;62(600):e949-957.

42. Blanchard CG, Ruckdeschel JC, Fletcher BA, Blanchard EB. The impact of oncologists' behaviors on patient satisfaction with morning rounds. Cancer. 1986:58(2):387-93.

43. Ong LM, Visser MR, Lammes FB, de Haes JC. Doctor-patient communication and cancer patients' quality of life and satisfaction. Patient Educ Couns. 2000;41(2):145-56

44. Zandbelt LC, Smets EM, Oort FJ, Godfried MH, de Haes HC. Satisfaction with the outpatient encounter: a comparison of patients' and physicians' views. J Gen Intern Med. 2004;19(11):1088-95.

45. Pot AM, van Dyck R, Deeg DJ. Perceived stress caused by informal caregiving. Construction of a scale. Tijdschr Gerontol Geriatr. 1995;26(5):214-9.

46. Higginson IJ, Evans CJ, Grande G, Preston N, Morgan M, McCrone P, Lewis P, Fayers $P$, Harding R, Hotopf $M$, et al. Evaluating complex interventions in end of life care: the MORECare statement on good practice generated by a synthesis of transparent expert consultations and systematic reviews. BMC Med. 2013;11:111.

47. Zimmermann C, Swami N, Krzyzanowska M, Hannon B, Leighl N, Oza A, Moore M, Rydall A, Rodin G, Tannock I, et al. Early palliative care for patients with advanced cancer: a cluster-randomised controlled trial. Lancet. 2014; 383(9930):1721-30.

48. Hennemann-Krause L, Lopes AJ, Araujo JA, Petersen EM, Nunes RA. The assessment of telemedicine to support outpatient palliative care in advanced cancer. Palliat Support Care. 2015;13(4):1025-30.

49. Hui D, Shamieh O, Paiva CE, Khamash O, Perez-Cruz PE, Kwon JH, Muckaden MA Park M, Arthur J, Bruera E. Minimal clinically important difference in the physical, emotional, and total symptom distress scores of the Edmonton symptom assessment system. J Pain Symptom Manag. 2016;51(2):262-9.

50. Cheung WY, Le LW, Zimmermann C. Symptom clusters in patients with advanced cancers. Support Care Cancer. 2009;17(9):1223-30.

51. Hauser W, Hansen E, Enck P. Nocebo phenomena in medicine: their relevance in everyday clinical practice. Dtsch Arztebl Int. 2012;109(26):459-65.

\section{Submit your next manuscript to BioMed Central and we will help you at every step:}

- We accept pre-submission inquiries

- Our selector tool helps you to find the most relevant journal

- We provide round the clock customer support

- Convenient online submission

- Thorough peer review

- Inclusion in PubMed and all major indexing services

- Maximum visibility for your research

Submit your manuscript at www.biomedcentral.com/submit 